\title{
Prospective evaluation of Quasistatic Ultrasound Elastography (USE) compared with Baseline US for parotid gland lesions: preliminary results of elasticity contrast index (ECI) evaluation
}

\author{
Vito Cantisani ${ }^{1}$, Emanuele David ${ }^{2}$, Armando De Virgilio ${ }^{3}$, Paul S Sidhu ${ }^{4}$, Hektor Grazhdani ${ }^{5}$, \\ Antonio Greco ${ }^{3}$, Marco De Vincentiiis ${ }^{3}$, Alessandro Corsi ${ }^{6}$, Flaminia De Cristofaro ${ }^{1}$, Luca \\ Brunese $^{7}$, Fabrizio Calliada ${ }^{8}$, Alfredo Blandino ${ }^{2}$, Mario Tombolini ${ }^{3}$, Giorgio Ascenti ${ }^{2}$, \\ Roberto Stramare ${ }^{9}$, Marcello Caratozzolo ${ }^{1}$, Ferdinando D'Ambrosio ${ }^{1}$
}

${ }^{1}$ Department of Radiology, Oncology, and Anatomy Pathology, Policlinico Umberto I, University Sapienza, Rome, Italy, ${ }^{2}$ Department of Biomedical Sciences and Morphological and Functional Imaging, University of Messina, Policlinico "G. Martino", Messina, Italy, ${ }^{3}$ Department of Sensory Organs, ENT Section, "Sapienza” University of Rome, Rome, Italy, ${ }^{4}$ Department of Radiology, King's College Hospital, Denmark Hill, London, UK, ${ }^{5}$ Associazione dei Cavalieri Italiani Sovrano Militare Ordine di Malta; Poliambulatorio Roma Eur, Rome, Italy, ${ }^{6}$ Department of Molecular Medicine, Policlinico Umberto I, University Sapienza, Rome, Italy, ${ }^{7}$ Department of Medicine and Health Sciences, University of Molise, Campobasso,Italy, ${ }^{8}$ University Hospital of Pavia, Pavia, Italy, ${ }^{9}$ University of Padova , Department of Medicine, Padova, Italy

\begin{abstract}
Aims: To assess the diagnostic value of elasticity contrast index (ECI) elastography, in comparison with conventional ultrasound for the differentiation of benign and malignant parotid lesions. Material and methods: Sixty-three consecutive patients with parotid gland tumors, treated surgically at a single tertiary center were enrolled. Ultrasound evaluation consisted of B-mode, color-Doppler and quasistatic ultrasound elastography (USE), conducted with the ECI technique. For each lesion the echogenicity, margins, vascularization, and capsulation were considered to determine benign or malignant lesions. Histology was considered the gold standard. Results: Using conventional parameters, the observer suggested malignant lesions in 20 cases and benign lesions in 43 cases (accuracy: 61.8\%). ECI $>3.5$ alone was the most accurate parameter (accuracy: 90.5\%), with sensitivity of $93.7 \%$ and specificity of $89.4 \%$. However, when combined with the other US criteria, no statistically significant diagnostic accuracy improvement was achieved. Conclusions: Our results show that USE with ECI index measurement can help to discriminate preoperatively benign from malignant lesions, with the exception of pleomorphic adenoma which is stiff. We suggest that USE can be used as an additional tool to conventional US evaluation of salivary gland lesions.
\end{abstract}

Keywords: parotid, ultrasound, elastography, parotidectomy, elastosonography

\section{Introduction}

Pre-operative differentiation between benign and malignant tumors is crucial in parotid gland surgery

Received 05.09.2016 Accepted 20.10.2016

Med Ultrason

2017, Vol. 19, No 1, 32-38

Corresponding author: Vito Cantisani, $\mathrm{MD}, \mathrm{PhD}$

Department of Radiology, Oncology, and Anatomy Pathology, Policlinico Umberto I, University Sapienza,

Viale del Policlinico 155, 00161, Rome, Italy

Phone: +39-0649976802, Fax:+39-0649976803

E-mail: vito.cantisani@uniroma1.it since the chosen surgical technique depends not only on the location of the tumor but also on the histologic type [1-4]. When a tumor is malignant, the total parotidectomy is indicated but in these cases the facial nerve may be resected or damaged while in benign neoplasms, which mainly involves the superficial lobe, a 'limited' or preneural parotidectomy is considered appropriate. An inaccurate preoperative diagnosis renders the informed consent description and discussion difficult, as the documentation of patient's willingness to be placed at risk for post-surgical complications such as facial nerve is crucial. Furthermore misdiagnosis could result in both 
hazardous under-treatment, and potentially avoidable overtreatment with permanent facial static and dynamic disfigurement. Fine-needle aspiration cytology (FNAC) guided by ultrasound (US) imaging is widely used to evaluate both neoplastic and inflammatory lesions of the salivary glands, albeit with variable success; sensitivity ranges from $57 \%$ to $98 \%$, specificity from $56 \%$ to $100 \%$, and accuracy from $78 \%$ to $98 \%$ [5-16] which also depends on the expertise and experience of the pathologist. FNAC is obtained in all salivary gland nodules, and normally performed in reference centers dealing with parotid gland tumors. Some authors support the role of cytology only in selected groups while others state that pre-surgical FNAC does not impact on the surgical handling of these lesions [7,17], highlighting the importance of preoperative imaging in surgical planning $[8,18,19]$.

High resolution US and magnetic resonance (MR) examinations are the primary imaging techniques for the evaluation of lesions of the major salivary glands. As parotid lesions are localized in the superficial lobe in $>90 \%$ of cases, it would seem that US, as a readily available, cost-effective and harmless technique, is the examination of choice. Moreover the application of parametric US techniques, including color-Doppler, US contrast agents and more recently, ultrasound elastography (USE) for assessing the lesion stiffness/elasticity characteristics, has been advocated to be useful for differentiating between different types of salivary gland lesions [20,21]. MR imaging is usually the second level imaging modality requested and the MR imaging findings of salivary gland tumors have been previously described [22-27]. General MRI features signifying malignancy are irregular margins, extra-glandular infiltration, perineural spread, and secondary lymphadenopathy [18] with low signal intensity and irregular lesion margins on T2-weighted MR images $[22,23]$. However, the reported sensitivities and specificities of these findings are low with considerable overlap [20,21,26,27].

USE is a promising technique for diagnostic imaging and has potential for differentiating benign from malignant lesions in the parotid gland. USE has also been proven to be useful in differentiating between noninvasive benign and malignant lesions in other organs [28-32].

The aim of this study was to examine whether the combination of conventional US imaging with USE improves the evaluation of parotid gland lesions and to determine which cut-off elasticity value would be the ideal for the differentiation between benign and malignant lesions. Furthermore, we pursued a secondary goal: to determine the elasticity of various benign tumors.

\section{Materials and methods}

Prospective analysis of salivary gland tumors by the means of B-mode US and real-time qualitative and quantitative USE was performed. The examined group consisted of 65 consecutive patients with parotid gland tumors, treated surgically in a single tertiary center, 29 female and 36 male subjects, aged 18-84 years (median $=56$ years). All subjects gave written informed consent. The research was carried out in accordance to the ethical guidelines of the Declaration of Helsinki and approved by the Department Board.

Evaluation was performed by means of B-mode US, color-Doppler US (CDUS) and quasistatic USE using ACCUVIX A30, RS 80 A (Samsung Medison Co. Ltd., Seoul, Korea). USE was conducted by Elastoscan ${ }^{\mathrm{TM}}$ software (Samsung Medison, Korea) installed in the same US unit. All evaluation was operated by an experienced radiologist who was blinded to clinical, cytological or histological characteristics. The study was carried out with a high frequency (10-18 MHz) linear US transducer, placed gently on patient's neck in hyperextension, while lying in supine position, with axial and longitudinal scanning. For each lesion we considered the following criteria: echogenicity, margins, vascularization, and capsule definition. Signs of possible benignity were considered the homogeneous hypoechogenicity or isoechogenicity, regular well defined margins with an eventual capsule, and slight vascularization. Signs indicating of malignancy were dis-homogeneous internal echogenicity, irregular or spiculated margins with no capsule, haphazard irregular and/or intense intra-nodular vascularization. The radiologic volume of each tumor was assessed (using the formula: $\mathrm{p} / 6 \mathrm{x}$ height $\mathrm{x}$ length $\mathrm{x}$ width). For elastography with elasticity contrast index (ECI) evaluation, the patient was asked to perform a breath hold for 3-4 seconds to allow the acquisition. No external compression with the transducer was needed as Elastoscan ${ }^{\mathrm{TM}}$ uses the pulsations of the carotid artery (in vivo compression) for the elastography response. The operator performed the examination in longitudinal planes and then drew the region of interest (ROI) to include the nodule only. Within the ROI the software assessed the elasticity contrast index ECI, a semiquantitative stiffness evaluation.

On the elastograms (placed on the left side of the screen, side by side with the B-mode image) the nodules and the parotid were color coded depending on their stiffness with a colorimetric scale on the screen indicating the corresponding hard and soft areas, color coded red and blue respectively. The largest diameter of the suspicious lesion was included in the image. A ROI as described above was manually placed by the operator. Elastogram 
was considered optimal when the screen quality indicator turned to green (multiple boxes that change color from red, to yellow or green). Then the ECI value was computed and displayed on the screen. A high ECI value index indicated that the parotid nodule was hard and presumably malignant [32].

All patients underwent FNAC, thereafter surgery, within one month of USE examination. Histopathology was available in all patients.

\section{Statistical analysis}

Statistical analysis was performed by means of Spearman's rank correlation coefficient for the age analysis, Kruskal-Wallis Test for more than two variables (concerning histology) and Mann-Whitney U Test for two variables (benign/malignant tumor's correlation). Cutoff values for continuous variables were calculated by receiver operation characteristic (ROC) analysis. Likelihood ratios were presented. The best cut-off point was defined by the highest sensitivity and specificity.

\section{Results}

We excluded 2 patients from this study because we could not perform a reliable elastographic examination due to excessive carotid artery pulsations. All the remaining patients were successfully evaluated and they underwent a superficial parotidectomy in cases of suspected benign tumor $(n=49)$ and a total parotidectomy in case of presumed malignant tumors, with $(\mathrm{n}=5)$ or without neck dissection $(\mathrm{n}=9)$ depending on the histological subtype and tumor extension. Delayed neck dissection $(\mathrm{n}=7)$ was

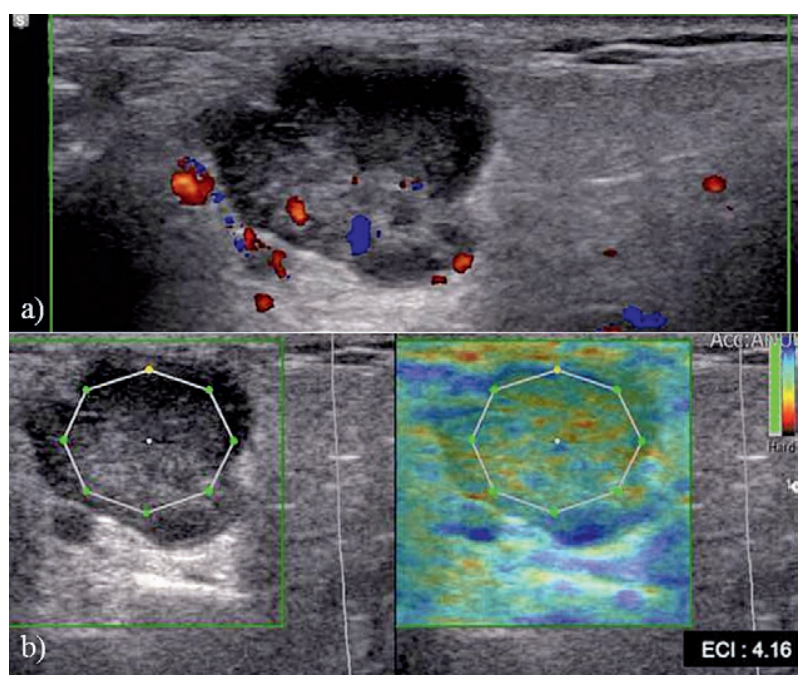

Fig 1. a) Iso/hypoechoic lesion with polycyclic margin, posterior acoustic enhancement, and some peripheral and internal spots at color Doppler US; b) The lesion presents high value of ECI at elastography evaluation performed on 20 days average following surgery in case of misdiagnosed malignant tumor. Adjuvant radiotherapy and chemotherapy were administered according to the 'National Comprehensive Cancer Network' (NCCN) guidelines.

The smallest diameter of the parotid lesion was of 6 $\mathrm{mm}$ and the largest was of $57 \mathrm{~mm}$. The median tumor volume was of $5.04 \mathrm{~cm}$.

Histology results showed 47 patients with benign and 16 patients with malignant tumors: pleomorphic adenoma ( $\mathrm{n}=18$ ) (fig 1), Warthin tumor ( $\mathrm{n}=17)$ (fig 2), mucoepidermoid carcinoma $(n=5)$, oncocytoma $(n=5)$, malignant lymphoma $(n=3)$, lymphoepithelial cyst $(n=3)$, ductal

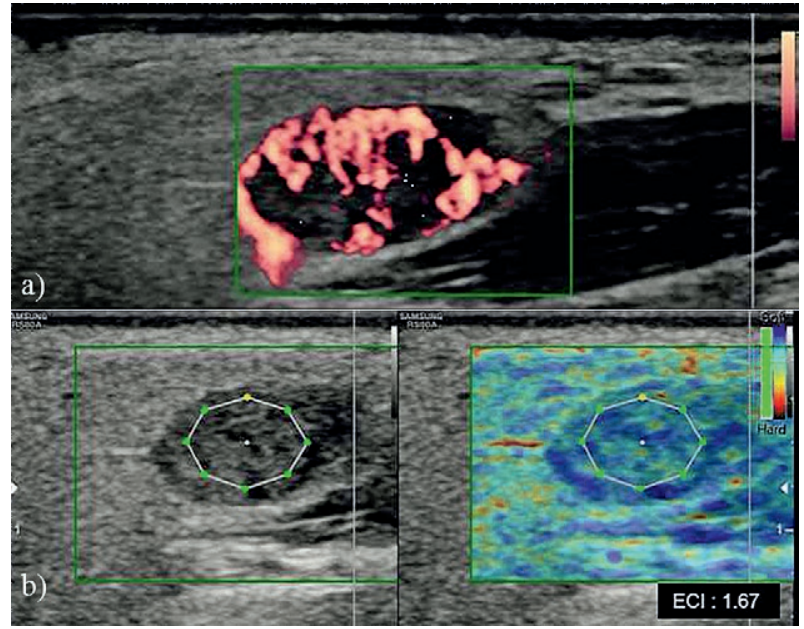

Fig 2. a) Hypoechoic oval shaped lesion with diffuse intra and perinodular vascularization; b) It shows low ECI value

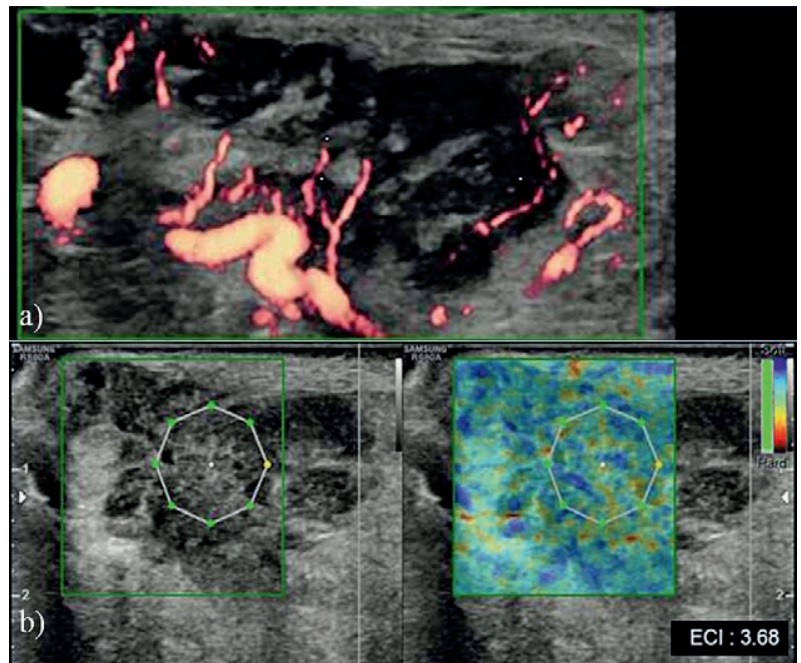

Fig 3. a) Irregular hypoechoic nodule with diffuse peripheral vascularity at power-Doppler Ultrasound; b) It presents high value of ECI suggestive of malignancy. 
cyst $(n=2)$, benign vascular tumor $(n=2)$, squamous cell carcinoma $(n=2)$, acinic cell carcinoma $(n=2)($ fig 3$)$, salivary duct carcinoma $(\mathrm{n}=2)$, and squamous cell carcinoma metastasis $(\mathrm{n}=2)$.

Using conventional parameters only, the observer suggested a malignant lesion in 20/63 (31.7\%) cases and benign in 43/63 (68.3\%) cases (accuracy: 61.8\%).

ECI calculated for each lesion is shown in Table I. Benign tumors showed a median ECI of 2.31 (range 1.1-4.43), whereas the malignant lesions had a median ECI of 5.55 (range 2-7.58). ROC analysis demonstrated that an ECI value of $>3.5$ was the ideal cut-off point for diagnosis of malignancy. Of note, the pleomorphic adenomas showed high ECI values: median of 3.12 (range 2.2-4.43), ECI values being higher than the cut-off value in $3 / 18(16.7 \%)$ cases. However in all 3 cases, at least two other US parameters suggested benign disease. The other common benign tumor, the Warthin tumor, was soft in all cases.

The diagnostic performance of each individual US criteria, ECI, and ECI combined with each individual US criteria, was statistically calculated and presented in Table II. The best result was found when ECI performed alone (the most accurate parameter), since with a cut-off value of ECI $>3.5$ the accuracy was $90.5 \%$, sensitivity of

Table I. The histopathology of the surgical specimens and the ECI values in the examined group.

\begin{tabular}{lllll}
\hline & \multicolumn{5}{c}{ ECI } & & \\
\hline & No. & Median & Range & SD \\
\hline All tumors & 63 & 3.13 & $1.1-7.58$ & 1.75 \\
Malignant (all) & 16 & 5.55 & $2-7.58$ & 1.53 \\
Benign (all) & 47 & 2.31 & $1.1-4.43$ & 0.80 \\
Pleomorphic adenoma & 18 & 3.12 & $2.2-4.43$ & 0.73 \\
Warthin tumor & 17 & 1.87 & $1.1-2.6$ & 0.41 \\
Other benign lesions & 12 & 1.71 & $1.3-2.4$ & 0.34 \\
\hline
\end{tabular}

No.: number of tumors, SD: standard deviation.
$93.7 \%$, and specificity of $89.4 \%$. When combined with other US criteria ECI showed a relatively minor diagnostic performance. FNAC was not diagnostic in $12 / 63$ cases $(19.0 \%)$ and in $8 / 63(12.7 \%)$ cases the FNAC diagnosis did not correspond to the final histology: FNAC suggested a benign lesion when a malignant tumor was the surgical and histology result. FNAC sensitivity, specificity, predictive positive value, negative predictive value and accuracy calculated on 51 patients were $33.3 \%, 100 \%$, $100 \%, 82.9 \%$, and $86.2 \%$ respectively.

\section{Discussions}

USE is a sonographic technique that assesses the tissue stiffness. As malignant tissue is generally stiffer than benign tissue, therefore, interest has been recently focused on the ability of USE as an aid in the differential diagnosis between benign and malignant salivary gland lesions. The technique has already gained attention for the lesions of the head and neck region [33-35] with promising results, particularly for thyroid lesions [36].

However, there is limited evidence for the usefulness of USE in the differential diagnosis of parotid tumors [21,37-40]. A typical sonoelastographic pattern for pleomorphic adenomas could not be demonstrated by Dumitriu et al [21]. Although it has been proposed that a semiquantitative 4-point elastographic score, adapted from a breast elastography score [39] can be used in parotid gland lesions, our experience suggests that such a scoring system is inadequate to classify and distinguish benign from malignant masses as both malignant and benign parotid tumors are in general markedly heterogeneous. For example, both mucoepidermoid and adenoid cystic carcinoma (which are the most frequent parotid malignant tumors) are, in the majority of cases, formed by a mixture of stiff and soft tissues. For this reason the maximum score of 4 is rarely encountered in the parotid malignancies.

Table II. The diagnostic performance of US criteria

\begin{tabular}{|c|c|c|c|c|c|c|c|}
\hline Parameter & $\begin{array}{l}\text { sensitivity \% } \\
\text { (CI) }\end{array}$ & $\begin{array}{l}\text { specificity \% } \\
\text { (CI) }\end{array}$ & $\begin{array}{l}\text { PPV \% } \\
\text { (CI) }\end{array}$ & $\begin{array}{l}\text { NPV \% } \\
\text { (CI) }\end{array}$ & $\begin{array}{l}\text { pos LR } \\
\text { (CI) }\end{array}$ & $\begin{array}{l}\text { neg LR } \\
\text { (CI) }\end{array}$ & $\begin{array}{l}\text { Accu- } \\
\text { racy } \\
\%\end{array}$ \\
\hline Echogenicity & $100(0.75-1)$ & $29.8(0-17-0.45)$ & $32.6(0.20-0.47)$ & $100(0.73-1)$ & $1.42(1.18-1.71)$ & 1 & 47.6 \\
\hline Vascularization & $87.5(0.60-0.97)$ & $51(0.36-0.65)$ & $37.8(0.22-0.55)$ & $92.3(0.73-0.98)$ & $1.78(1.26-2.52)$ & $0.24(0.06-0.93)$ & 60.3 \\
\hline Margins & $50(0.25-0.74)$ & $97.8(0.87-0.99)$ & $88.8(0.50-0.99)$ & $85.2(0.72-0.92)$ & $23(3.18-173.63)$ & $0.51(0.31-0.83)$ & 85.7 \\
\hline Capsulation & $100(0.75-1)$ & $38.3(0.24-0.53)$ & $35.5(0.22-0.51)$ & $100(0.78-1)$ & $1.62(1.29-2.03)$ & 1 & 53.9 \\
\hline $\mathrm{ECI}(>3.5)$ & $93.7(0.67-0.99)$ & $89.4(0.76-0.96)$ & $75(0.50-0.90)$ & $97.7(0.86-0.99)$ & $8.81(3.81-20.37)$ & $0.06(0.01-0.46)$ & 90.5 \\
\hline ECI + echogenicity & $100(0.75-1)$ & $25.5(0.14-0.40)$ & $31.480 .19-0.46)$ & $100(0.69-1)$ & $1.34(1.13-1.58)$ & 1 & 44.4 \\
\hline ECI + vascularization & $100(0.75-1)$ & $44.7(0.30-0.59)$ & $38.1(0.23-0.54)$ & $100(0.80-1)$ & $1.80(1.39-2.33)$ & l & 58.7 \\
\hline $\mathrm{ECI}+$ margins & $93.7(0.67-0.99)$ & $87.2(0.73-0.94)$ & $71.4(0.47-0.87)$ & $97.6(0.85-0.99)$ & $7.34(3.44-15.67)$ & $0.07(0.01-0.48)$ & 88.9 \\
\hline $\mathrm{ECI}+$ capsulation & $100(0.75-1)$ & $29.8(0.17-0.45)$ & $32.6(0.20-0.47)$ & $100(0.73-1)$ & $1.42(1.18-1.71)$ & / & 47.6 \\
\hline
\end{tabular}

CI: confidence interval, 
Bhatia et al [37] assessed the parotid tumors by measuring the elasticity in a purely quantitative way $(\mathrm{kPa}, \mathrm{m} /$ $\mathrm{sec}$ at shear wave elastography) and found a significant difference between pleomorphic adenomas and Warthin tumors elasticity quantitative values. However, the authors observed little benefit in the benign-malignant differentiation of parotid tumors, particularly as pleomorphic adenoma quantitative values were similar and overlapping with malignant tumors. In particular, the observed intralesional variability of stiffness, which in pleomorphic adenoma can be high due to the tissue heterogeneity, led to the conclusion that elastography in parotid glands is unsuitable for excluding malignancy [37].

Using the same technique of shear wave elastography in 43 parotid tumors, another group found good performance in parotid gland lesions [39]; the mean elasticity values, in $\mathrm{kPa}$, differed significantly between the lesions and the normal parenchyma and furthermore, differed significantly between the benign and malignant lesions. A meta-analysis published in 2015 [40] considered 9 eligible studies with no publication bias on USE for salivary gland masses benign-malignant differentiations, with a total of 581 lesions, and found good performance with a pooled sensitivity and specificity of $76 \%(95 \%$ confidence interval $[\mathrm{CI}])$ and $73 \%(95 \% \mathrm{CI})$ respectively. The results showed that the malignant lesions were commonly stiffer than the benign ones, but there was still a substantial overlap between the elastographic properties of malignant and benign parotid lesions. The authors suggested that USE can be used as an adjunct to conventional US for the evaluation of salivary glands, however its overall accuracy cannot confidently obviate, in the routine clinical practice, the need of biopsy. All these studies used the traditional form of elastography, the free-hand compression USE, which is an operator-dependent technique, with the drawback that the amount of the applied external pressure, affects the tissue elasticity detected. We used the quasistatic USE with the compression detected and measured from the carotid artery pulsations, possibly a more accurate technique.

In the present study, for the first time we evaluated USE with a quasistatic technique (Elastoscan ${ }^{\mathrm{TM}}$ ) in which a semiquantitative evaluation by means of ECI index is used to differentiate parotid tumors. In this technique a single superimposed ROI included the maximum lesional tissue possible and assessed the tissue stiffness at carotid pulsations. A statistically significant difference between benign and malignant tumor mean elasticity $(p<0.001)$ was observed. The ECI cut-off value of 3.5 for the differential diagnosis between benign and malignant tumor considered alone was superior in terms of sensitivity, specificity, and accuracy to the other US pa- rameters, except for echogenicity and capsule definition which were more sensitive but less specific. Whereas, when combined with the other US criteria, ECI showed an overall minor diagnostic performance. Diagnostic performance was the lowest with the B-mode features (accuracy 61.8\%). FNAC was found to be less accurate in comparison with B-mode, color Doppler US, and USE with a high percentage of non-diagnostic results (19\%) and low sensitivity (33.3\%).

In our experience, on B-mode benign tumors are usually well-defined, often with a typical homogeneity, may contain calcification, and are well or sparsely vascularized. They may also show features suggesting specific abnormalities, such as sharp borders, lobulated contours, posterior acoustic enhancement in pleomorphic adenoma, cystic areas in Warthin tumors, calcifications representing phleboliths in hemangiomas, or oval and hypoechoic appearance with hyperechoic linear structures in lipomas. Regarding malignant lesions, characteristic features are hypoechoic inhomogeneous structure, irregular and poorly defined margins, local invasion into surrounding soft tissues, and pathologic lymph nodes.

A peculiar finding was the fact that pleomorphic adenoma, a benign tumor, showed a high ECI value: median of 3.12 (range 2.2-4.43) and thus proved to be difficult to differentiate from malignancy, with overlapping ECI values. In our series ECI values were high in pleomorphic adenomas, in 3 cases higher than the cutoff value; however in all these 3 cases, at least two US parameters suggested a benign disease. On the contrary, the other common benign tumor, the Warthin tumor, was soft, with a median of ECI of 1.87 (range 1.1-2.6) and we concluded that the ECI value may be a helpful finding in differentiating the pleomorphic adenoma from the Warthin tumor when the result of FNAC is inconclusive. In our 6 case series that were diagnosed preoperatively as Warthin tumor using FNAC, while the final pathological reports documented pleomorphic adenoma; in only $2 / 6$ of cases pleomorphic adenoma diagnosis could be heavily suspected based only on US findings, whereas USE made the correct diagnosis of pleomorphic adenoma with a median ECI value of 2.9. Further studies should investigate the value of ECI in differentiating Warthin's tumor from pleomorphic adenoma especially in the cases in which baseline US or FNAC is not valuable enough to differentiate between the two tumors.

We have to mention as a limitation that the ECI values may slightly differ according to the strength of the pulsation from the carotid artery depending on age, atherosclerosis, and hypertension. Due to this issue, 2 patients were excluded from this study because we could not perform a reliable elastographic examination due to 
excessive carotid artery pulsations. Another bias was represented by the fact that only one expert and experienced USE operator performed the examination and the interobserver or intra-observer variability were not assessed. Furthrmore, it is known that cystic lesion in the thyroid yield false results in the USE evaluation [28,29,35,41]. However in our cohort, cystic lesions in the parotid lesions were rare, and therefore we could not statistically evaluate this known confounding factor on ECI.

As a conclusion of these preliminary results we can mention that our study showed that USE with ECI index measurement improves the sensitivity of US for discriminating preoperatively benign from malignant parotid lesions. However, the user should be aware that pleomorphic adenomas are stiffer than other benign lesions. Therefore, we suggest the use of USE as an additional tool to conventional US evaluation in the diagnosis of salivary gland lesions. Larger and multi-center studies can better define the value of USE with ECI evaluation for parotid masses.

Conflict of interest: The authors declared the following potential conflicts of interest with respect to the research, authorship, and/or publication of this article: Vito Cantisani was formerly a lecturer for Bracco, Toshiba, and Samsung. Fabrizio Calliada was formerly a lecturer for Bracco, Toshiba, and Mindray. Paul Sidhu lectured for Bracco, Siemens, Hitachi, and GE.

Acknowledgment and guarantor, our esteemed director: Prof. Ferdinando D'Ambrosio Department of Radiology, Oncology, and Anatomy Pathology, Policlinico Umberto I, University Sapienza, Viale del Policlinico 155, 00161, Rome, Italy.

\section{References}

1. Yu GY, Ma DQ, Liu XB, Zhang MY, Zhang Q. Local excision of the parotid gland in the treatment of Warthin's tumour. Br J Oral Maxillofac Surg 1998;36:186-189.

2. Carlson GW. The salivary glands. Embryology, anatomy, and surgical applications. Surg Clin North Am 2000;80:261273.

3. Magnano M, Gervasio CF, Cravero L, et al. Treatment of malignant neoplasms of the parotid gland. Otolaryngol Head Neck Surg 1999;121:627-632.

4. Rehberg E, Schroeder HG, Kleinsasser O. Surgery in benign parotid tumors: individually adapted or standardized radical interventions? Laryngorhinootologie 1998;77:283288.

5. Frable MA, Frable WJ. Fine needle aspiration biopsy of salivary glands. Laryngoscope 1991;101:245-249.
6. Stewart CJ, MacKenzie K, Mcgarry GW, Mowat A. Fineneedle aspiration cytology of salivary gland: a review of 341 cases. Diagn Cytopathol 2000;22:139-146.

7. Cohen EG, Patel SG, lin O, et al. Fine-needle aspiration biopsy of salivary gland lesions in a selected patient population. Arch Otolaryngol Head Neck Surg 2004;130:773-778.

8. Zurrida S, Alasio 1, Tradati N, Bartoli C, Chiesa F, Pilotti S. Fine needle aspiration of parotid masses. Cancer 1993;72:2306-2311.

9. Jayaram G, Verma AK, Sood N, Khurana N. Fine needle aspiration cytology of salivary gland lesions. J Oral Pathol Med 1994;23:256-261.

10. Atula T, Greénman R, Laippala P, Klemi PJ. Fine-needle aspiration biopsy in the diagnosis of parotid gland lesions: evaluation of 438 biopsies. Diagn Cytopathol 1996; 153:185-190.

11. Cristallini EG, Ascani S, Farabi R, et al. Fine needle aspiration biopsy of salivary gland, 1985- 1995. Acta Cytol 1997;41:1421-1425.

12. Cajulis RS, Gokaslan ST, Yu GH, Frias-Hidvegi D. Fine needle aspiration biopsy of the salivary glands. A five-year experience with emphasis on diagnostic pitfalls. Acta Cytol 199741:1412-1420.

13. Al-Khafaji BM, Nestok BR, Katz Rl. Fine needle aspiration of 154 parotid masses with histologic correlation: ten-year experience at the University of Texas M.D. Anderson Cancer Center. Cancer 1998;84:153-159.

14. Que Hee CG, Perry CF. Fine-needle aspiration cytology of parotid tumours: is it useful? ANZ J Surg 2001;71:345-348.

15. Bajaj Y, Singh S, Cozens N, Sharp J. Critical clinical appraisal of the role of ultrasound guided fine needle aspiration cytology in the management of parotid tumours. J Laryngol Otol 2005;119:289-292.

16. Carrillo JF, Ramirez R, Flores 1, et al. Diagnostic accuracy of fine needle aspiration biopsy in preoperative diagnosis of patients with parotid gland mass. J Surg Oncol 2009;100:133-138.

17. Batsakis JG, Sneige N, el-Naggar AK. Fine-needle aspiration of salivary gland: its utility and tissue effects. Ann Otol Rhinol Laryngol 1992;101:185-188.

18. Layfield LJ, Tan P, Glasgow BJ. Fine-needle aspiration of salivary gland lesions: comparison with frozen sections and histologic findings. Arch Pathol Lab Med 1987;111:346353.

19. Takashima S, Takayama F, Wang Q, Kurozumi M, Sekiyama Y, Sone S. Parotid gland lesions: diagnosis of malignancy with MRI and flow cytometric DNA analysis and cytology in fine-needle as- piration biopsy. Head Neck 1999;21:43-51.

20. Bhatia KS, Rasalkar DD, Lee YP, et al. Evaluation of realtime qualitative sonoelastography of focal lesions in the parotid and submandibular glands: applications and limitations. Eur Radiol 2010;20:1958-1964.

21. Dumitriu D, Dudea SM, Botar-Jid C, Băciuţ G. Ultrasonographic and sonoelastographic features of pleomorphic adenomas of the salivary glands. Med Ultrason 2010;12:175183. 
22. Som PM, Biller HF. High-grade malignancies of the parotid gland: identification with MR imaging. Radiology 1989; 173: 823- 826.

23. Swartz JD, Rothman MI, Marlowe FI, Berger AS. MR imaging of parotid mass lesions: attempts at histopathologic differentiation. J Comput Assist Tomogr 1989;13:789-796.

24. Teresi LM, Lufkin RB, Wartham DG, Abemayor E, Hanafee WN. Parotid masses: MR imaging. Radiology 1987; 163:405-409.

25. Freling NJ, Molenaar WM, Vermey A, et al. Malignant parotid tumors: clinical use of MR imaging and histologic correlation. Radiology 1992;185:691-696.

26. Joe VQ, Westesson PL. Tumors of the parotid gland: MR imaging characteristics of various histologic types. AJR Am J Roentgenol 1994;163:433-438.

27. Mascaro L, Ferrari C, Grazioli L, Aragno D, Chiesa A. T2 relaxation of the parotid gland of patients affected by pleomorphic adenoma. Magn Reson Imaging 1999;17:723-730.

28. Cosgrove D, Piscaglia F, Bamber J, et al. EFSUMB guidelines and recommendations on the clinical use of ultrasound elastography. Part 2: Clinical applications. Ultraschall Med 2013;34:238-253.

29. Cantisani V, Maceroni P, D'Andrea V, et al. Strain ratio ultrasound elastography increases the accuracy of colour-Doppler ultrasound in the evaluation of Thy-3 nodules. A bi-centre university experience. Eur Radiol 2016;26:1441-1449.

30. Ferraioli G, Filice C, Castera L, et al. WFUMB guidelines and recommendations for clinical use of ultrasound elastography: Part 3: liver. Ultrasound Med Biol 2015;41:11611179.

31. Correas JM, Drakonakis E, Isidori AM, et al. Update on ultrasound elastography: miscellanea. Prostate, testicle, musculo-skeletal. Eur J Radiol 2013;82:1904-1912.

32. Cantisani V, Lodise P, Di Rocco G, et al. Diagnostic accuracy and interobserver agreement of Quasistatic Ultrasound
Elastography in the diagnosis of thyroid nodules. Ultraschall Med 2015;36:162-167.

33. Suh CH, Choi YJ, Baek JH, Lee JH. The diagnostic performance of shear wave elastography for malignant cervical lymph nodes: A systematic review and meta-analysis. Eur Radiol 2016 May 5. doi: 10.1007/s00330-016-4378-3.

34. Tatar IG, Kurt A, Hekimoglu B. Advantages and pitfalls of elastosonography in the evaluation of cervical lymph nodes. Med Ultrason 2013;15:333-334.

35. Cantisani V, Grazhdani H, Ricci P, et al. Q-elastosonography of solid thyroid nodules: assessment of diagnostic efficacy and interobserver variability in a large patient cohort. Eur Radiol 2014;24:143-150.

36. Cantisani V, Lodise P, Grazhdani H, et al. Ultrasound elastography in the evaluation of thyroid pathology. Current status. Eur J Radiol 2014;83:420-428.

37. Bhatia KS, Cho CC, Tong CS, Lee YY, Yuen EH, Ahuja AT. Shear wave elastography of focal salivary gland lesions: preliminary experience in a routine head and neck US clinic. Eur Radiol 2012;22:957-965.

38. Klintworth N, Mantsopoulos K, Zenk J, Psychogios G, Iro H, Bozzato A. Sonoelastography of parotid gland tumours: initial experience and identification of characteristic patterns. Eur Radiol 2012;22:947-956.

39. Wierzbicka M, Kaluzny J, Szczepanek-Parulska E, et al. Is sonoelastography a helpful method for evaluation of parotid tumors? Eur Arch Otorhinolaryngol 2013;270:21012107.

40. Li C, Zhang C, Li N, Li J. Compression Real-time Elastography for Evaluation of Salivary Gland Lesions: A Metaanalysis. J Ultrasound Med 2016;35:999-1007.

41. Cosgrove D, Barr R, Bojunga J, et al. WFUMB Guidelines and Recommendations on the Clinical Use of Ultrasound Elastography: Part 4. Thyroid. Ultrasound Med Biol. 2016 Aug 25. doi: 10.1016/j.ultrasmedbio.2016.06.022. 